A/H1N1 Vaccine Intentions in College Students: An Application of the Theory of Planned Behavior

Author Contact Information:

Vinita Agarwal, Ph.D. (2009, Purdue University)

Assistant Professor, Department of Communication Arts

FH 272, 1101 Camden Ave.

Salisbury University, Salisbury MD 21801

Email: vxagarwal@salisbury.edu

Tel: 410-677-0083/ Fax: 410-548-3002

Acknowledgments:

The author is grateful to the executive editor and two anonymous reviewers for their insightful comments and feedback that guided the development of the manuscript. The author thanks the Department of Communication, University of Louisville, Louisville, Kentucky for the support provided the study. Earlier versions of this paper were presented at the Kentucky Conference in Health Communication, 2010, the National Communication Association, 2011, and the Eastern Communication Association, 2012. 


\begin{abstract}
Objective: To test the applicability of the theory of planned behavior (TPB) in college students who have not previously received the A/H1N1 vaccine.

Participants: Undergraduate communication students at a metropolitan southern university.

Methods: In January-March 2010, students from voluntarily participating communication classes completed a hard-copy survey assessing TPB and clinically significant constructs. Hierarchical regression equations predicted variance in vaccine intentions of students who had not received a flu shot $(N=198,70 \%$ Caucasian $)$.
\end{abstract}

Results: The TPB model explained $51.7 \%(p<.001)$ of variance in vaccine intentions. Controlling for side effects, self-efficacy and perceived comparative susceptibility predicted intentions when entered in the first block, while attitudes, subjective norms, and perceived behavioral control significantly contribute when entered in the second block.

Conclusions: For students who have not previously received a flu vaccine, vaccine communication should utilize self-efficacy and perceived comparative susceptibility to employ the TPB to promote vaccine intentions.

Keywords: pandemic influenza A (H1N1) 2009 virus, A/H1N1 vaccine intentions, self efficacy, perceived comparative susceptibility, side effects, theory of planned behavior, college students 


\section{A/H1N1 Vaccine Intentions in College Students: An Application of the Theory of Planned Behavior}

Although the novel pandemic influenza A (H1N1) 2009 virus disproportionately impacted college students in the 2009-2010 flu season, surveillance data suggests only $8 \%$ of

5 college students accepted the A/H1N1 vaccine. ${ }^{1}$ Epidemiological studies indicate young adults up to 24 years of age in college settings are at a higher risk of contracting influenza. ${ }^{2}$ It is one of the objectives of the Healthy Campus_-2020 initiative of the American College Health Association (ACHA) to reduce the proportion of college students who report adverse performance from cold/flu/sore throat (from $18 \%$ in 2010 to $16.2 \%$ in 2020; AI-1.4, Question

$1045 \mathrm{~A} 7$ ) and increase the proportion of college students who receive the influenza vaccine each year (from $39.9 \%$ in 2010 to $43.9 \%$ in 2020; Question 40C, item IID-12). ${ }^{3}$

The pandemic influenza A (H1N1) 2009 virus, also known as the A(H1N1)pdm09 virus (A/H1N1) was identified in April 2009 as a genetically and antigenically distinct form of the influenza A virus subtype previously found in swine. ${ }^{4}$ The $\mathrm{A} / \mathrm{H} 1 \mathrm{~N} 1$ virus was declared a global

15 pandemic in June 2009. In the U.S., the Centers for Disease Control and Prevention's (CDC) 2009 H1N1 influenza vaccination campaign (A/H1N1 vaccine) was the primary public health initiative to address the health threat with a goal to increase vaccination uptake among those identified by epidemiological and virologic data to be at higher risk of contracting infection. ${ }^{5}$ The U.S. Public Health Emergency for 2009 H1N1 Influenza expired on June 23, 2010, when the

$20 \mathrm{~A} / \mathrm{H} 1 \mathrm{~N} 1$ influenza virus was classified as post-pandemic. However, the virus is expected to continue to behave as a seasonal influenza A virus in the post-pandemic stage, thus underscoring the need to continue to update pandemic surveillance and preparedness. ${ }^{6}$ 
Young adults are susceptible to health-compromising behaviors in part due to a heightened sense of invulnerability that lead them to ignore risks. ${ }^{7,8}$ Evidence of the optimistic

25 bias in college students has been well documented. For example, recent findings examining the A/H1N1 outbreak in Fall 2009 demonstrate that a sense of self-efficacy contributes to vaccine intentions in students and the relationship was moderated by comparative optimism after controlling for perceived risk. ${ }^{9}$ Further, studies from different national contexts suggest that while young adults may make changes in hygienic behavior in response to flu prevention

30 messages, ${ }^{10}$ barriers to vaccine acceptance in this population persist. These include factors such as a lack of perception of vaccination as an efficacious anti-epidemic measure, ${ }^{11}$ or a lack of perception of the A/H1N1 virus as a credible threat. ${ }^{12}$ In the U.S., despite the institutional availability of preventive vaccines such as the TIV, often free of charge for the seasonal flu, low levels of vaccine acceptance have been noted among students. ${ }^{13,14}$ Challenges faced by CDC's

35 vaccination programs include effective promotion of vaccination services to target audiences and getting providers and immunization programs to work together. ${ }^{15}$

The 2009—2010 flu season A/H1N1 monovalent inactivated vaccine (MIV) and the live, attenuated vaccine (LAMV) for the $\mathrm{A} / \mathrm{H} 1 \mathrm{~N} 1$ influenza virus were distinct from the seasonal trivalent inactivated (TIV) and live, attenuated (LAIV) influenza vaccines. ${ }^{16}$ The $2013-2014$ seasonal TIV includes the H1N1 strain and is made to protect against three flu viruses - the influenza $\mathrm{A}(\mathrm{H} 1 \mathrm{~N} 1)$ virus, an influenza $\mathrm{A}(\mathrm{H} 3 \mathrm{~N} 2)$ virus, and an influenza B virus, alongside an additional quadrivalent vaccine that protects against two influenza A viruses and two influenza B viruses. ${ }^{5}$ However, in order to be effective, vaccine communication needs to be tailored to audiences' immunization knowledge and beliefs. In addition to weighing vaccine characteristics, 45 individuals also consider vaccine perceptions and their likelihood of infection when weighing the 
acceptability of voluntary, preventive vaccines. ${ }^{17,18}$ For example, A/H1N1 vaccine-specific misconceptions such as fear of the live virus in the LAIV was confused with the A/H1N1 vaccine which has the inactivated (killed) virus which had a negative influence on vaccination acceptance. ${ }^{19}$ Other factors such as the influence of social networks supportive of vaccination ${ }^{20}$ and past influenza vaccine behaviors (e.g., having had a flu shot in the past 5 years) have also been found to influence vaccine acceptance ${ }^{21}$ and intentions. ${ }^{22}$

As a value-expectancy model incorporating attitudes, subjective norms, and perceived behavioral control to understand intentions and behavior, the theory of planned behavior (TPB) can provide a useful framework to examine vaccination intentions. ${ }^{23,24}$ The TPB has been used as

55 a theoretical framework to examine $\mathrm{A} / \mathrm{H} 1 \mathrm{~N} 1$ vaccination among different populations and contexts in a few studies globally. ${ }^{25-27}$ For example, support was found or the TPB model in predicting vaccination intentions among priority group adults in UK highlighting the need to target interventions to well-defined groups. ${ }^{28}$ More recently, support has also been found for augmented TPB models incorporating perceived susceptibility, ${ }^{29}$ anticipated emotions, ${ }^{30,31}$ and

60 self-efficacy ${ }^{32}$ in a range of contexts and populations, thus highlighting the need to further examine these variables in the TPB.

As the TPB has found support in a range of content domains and populations, ${ }^{33-35}$ by extending it to the domain of student voluntary vaccination acceptance, research can provide public health professionals with useful insights for reaching specific audiences. This study

65 extends the applicability of the TPB to understand $\mathrm{A} / \mathrm{H} 1 \mathrm{~N} 1$ vaccination intentions in college students who have not accepted the vaccine along with the contribution of self-efficacy and perceived comparative susceptibility. 


\section{Theory of Planned Behavior}

The TPB builds on the assumption that individuals can rationally evaluate their options and

70 beliefs associated with a behavior before formulating their intention to perform the behavior. ${ }^{36}$ Further, the TPB posits that behavioral intention, or the assessment of whether or not a person plans to perform a particular behavior, is the most important and immediate antecedent of the actual performance of that behavior (i.e., stronger intentions predict a greater likelihood of performing the behavior). According to the TPB, an individual's attitude toward the behavior,

75 subjective norms of behaviors, and perceived behavioral control toward the behavior are the three determinants predicting behavioral intentions. ${ }^{36}$

Attitude toward the behavior is an individual's positive or negative belief about performing a specific behavior, and is determined by the individual's beliefs about the consequences of performing the behavior (behavioral beliefs) alongside an evaluation of those consequences

80 (outcome evaluations). ${ }^{36}$ In general, an individual is more likely to perform a behavior if he or she has a positive attitude toward the behavior (e.g., student nurses' intention to get the influenza vaccine is predicted by their attitude toward the vaccine ${ }^{22}$ ).

Subjective norms are a function of beliefs that significant others (e.g., close friends or family) approve or disapprove of the behavior and are determined by one's normative beliefs

85 (i.e., the beliefs that underlie subjective norms) and motivation to comply with those beliefs. According to the TPB, an individual is more likely to perform a specific behavior, such as obtain a vaccine, when the individual perceives that important others hold positive beliefs about the vaccine, recommend getting vaccinated, or receive the vaccine. ${ }^{36}$ 
Perceived behavioral control comprises an individual's beliefs about the presence of factors

90 that may impede or facilitate their ability to perform the behavior. ${ }^{36}$ The conceptual domain of perceived behavioral control includes the components of self-efficacy (ease or difficulty of performing a behavior) and controllability (the extent to which the performance of the behavior is within the individual's volitional control). ${ }^{37}$ In order to understand the distinct contributions of self-efficacy and controllability these can be considered independently in the TPB model. ${ }^{37}$ As

95 self-efficacy has been noted as a salient factor in college students, ${ }^{32}$ the present research seeks to determine its specific contribution as well as the specific contribution of controllability (perceived behavioral control) in the TPB model.

The goal of the present study is to examine the applicability of the TPB taking into account the contribution of perceived comparative susceptibility in the $\mathrm{A} / \mathrm{H} 1 \mathrm{~N} 1$ vaccine-intentions of 100 college students who have not received the vaccine. The following hypotheses are tested:

H1a: Self-efficacy will negatively predict vaccination intentions controlling for perception of vaccine side effects and perceived comparative susceptibility such that greater self-efficacy will predict lower vaccination intentions.

H1b: Perceived comparative susceptibility will positively predict vaccination intentions 105 controlling for perception of vaccine side effects and self-efficacy such that greater perceived comparative susceptibility will predict higher vaccination intentions.

$\mathrm{H} 2 \mathrm{a}$ : Controlling for subjective norms and perceived behavioral control attitudes will positively predict vaccination intentions after accounting for vaccine side effects, self-efficacy, and perceived comparative susceptibility. 
$\mathrm{H} 2 \mathrm{~b}$ : Controlling for attitudes and perceived behavioral control, subjective norms will positively predict vaccination intentions after accounting for vaccine side effects, self-efficacy, and perceived comparative susceptibility.

H2c: Controlling for attitudes and subjective norms, perceived behavioral control will negatively predict vaccination intentions after accounting for vaccine side effects, self-efficacy,

115 and perceived comparative susceptibility.

\section{Methods}

\section{Setting, Population, and Recruitment}

The student sample was derived from self-selected undergraduate communication classes of a mid-size southern, metropolitan research university. The total university undergraduate

120 student body in Fall 2009-2010 academic year (AY) was 22,031. ${ }^{38}$ Undergraduate student enrollment demographics in 2009-2010 AY reflect a predominantly Caucasian student population with a roughly equal distribution of males and females (Males, $N=10,608$, Females, $N=11,423 ; 76.1 \%$ Caucasian, $11.1 \%$ African-American; total undergraduate enrollment, $N=$ $15,619)$ with undergraduate class ranking reflecting a slightly lower enrollment in the

125 Sophomore and Junior years (Freshman $N=4026$, Sophomore, $N=2,972$, Junior, $N=3,075$, and Senior, $N=4,130)$. A majority of the first-time Freshmen (59.2\%) reported living on campus and the university reported an overall $95 \%$ student housing occupancy rate. The university comprises 12 schools conferring degrees in Law, Business, Arts and Sciences, Dentistry, Social Work, Medicine, Music, Engineering, and Public Health, among others. ${ }^{38}$ 
An email was sent to faculty members of the communication department soliciting study participation of students in return for extra credit in class. As communication students study media messages and effects, the study participants can be considered to be more aware of media

135 messages and goals than other majors. Participating classes ranged from senior-level public relations (advanced public relations) to introductory classes (introduction to mass communication), research methods, and special topics (health communication) courses. Thus participants ranged from freshmen, sophomores, juniors, and seniors (Table 1). Students who were not interested in participating in the survey were provided an alternative extra credit

140 opportunity. The university institutional review board approved the study.

Participant recruitment was conducted by the researcher providing an in-person introduction to the study in the participating classes before hard copy survey administration. Because of the high media coverage and awareness of the $\mathrm{A} / \mathrm{H} 1 \mathrm{~N} 1$ virus during the datagathering period, the introduction explained that communication students were participating in

145 the study to understand the role of individual factors in student intention to obtain the A/H1N1 vaccine. Additional surveys were also given to instructors to hand out to students who were absent or otherwise unable to obtain the survey. Students who were interested in participating in the study were given the option to return the completed surveys at the end of two weeks during a class announcement and sign their name on a sign-up sheet in return for extra credit in the class.

150 Participants could also drop the survey in a box outside the researcher's office with a sign-up sheet for receiving class credit. In that case, the researcher emailed the completed list to the class instructor. Because a mass email for participant recruitment was not sent out to the entire student population, a response rate cannot be determined. However, the sample participant demographics show these are reflective of the undergraduate population at the university. 


\section{Measures}

The TPB constructs of attitudes, subjective norms, and perceived behavioral control, and behavioral intention toward the A/H1N1 vaccine were adapted from Ajzen's TPB questionnaire. ${ }^{23}$ The construct of perceived comparative susceptibility was adapted from McQueen et al. (2008) ${ }^{39}$ and vaccine side effects and self-efficacy were adapted from Chapman

160 et al. (1999). ${ }^{40}$ Unless otherwise mentioned, all variables were averaged on $1-5$ Likert scale (1 $=$ the lowest value of the construct and $5=$ the highest). Items that were phrased in the semantically opposite direction (i.e., $5=$ lowest value of construct and $1=$ highest value of construct) were reverse-coded such that the higher value of the construct measured the higher numerical choice on the item response set. Once all items for a scale were in the same direction, 165 the items were averaged to create the scale.

\section{Demographic Characteristics}

Participants were asked to report their age, sex, ethnicity, class ranking, and whether they knew the university was offering a free $\mathrm{A} / \mathrm{H} 1 \mathrm{~N} 1$ vaccine (Table 1).

\section{Psychological Measures}

Participants reported their attitudes, subjective norms, perceived behavioral control, perceived comparative susceptibility, self-efficacy, and behavioral intention on 5-point intervallevel Likert scales.

\section{Analyses}

Frequencies and other descriptive statistics were used to describe the sample. Factor 175 analysis utilizing principal components analysis (PCA) framework with Varimax rotation was 
employed to assess the proportion of variance explained by the factor in the data. Cronbach's alpha coefficient was examined to assess internal consistency of items toward measuring the construct. Participants who marked "No" to the binary question: "Have you ever received a flu shot" $($ Yes $=1 ;$ No $=2)$ were selected into the data file used for the present study. Hierarchical multiple regression equations were set up to test the TPB model. The statistical software IBM SPSS Statistics 21 was used for data analysis.

\section{Results}

\section{Sample Description}

Table 1 reports the study participant characteristics of all participants and the study

185 sample of students who did not receive a flu shot. From the parent data file, those who marked "No" to the condition "Have you ever received a flu shot?" $(N=198 ; 39.6 \%)$ were selected to a new dataset that was employed for the present analyses (94\% between $18-25$ years, $70 \%$ Caucasian, 21\% African-American; 58\% female). The study sample characteristics are reflective of the population of undergraduate enrollment at the university.

\section{Univariate Analyses}

Attitude. Attitude was defined as “a person's overall evaluation of performing the behavior in question," 23 and included the items: "Getting the H1N1 vaccine from my university will be beneficial for me $(\lambda=.93)$, " "Getting the H1N1 vaccine from my university will be painful" (reverse coded), "Getting the H1N1 vaccine from my university will be good for me $(\lambda$ $195=.92)$," "Getting the H1N1 vaccine from my university is not important for me $(\lambda=.64)$ " (reverse coded), and "Getting the H1N1 vaccine from my university will be useful for me $(\lambda=$ .89)." All items loaded satisfactorily, with the factor loading of one item being acceptable (H1N1 
vaccine is important to me, $\lambda=.64$ ). One item ("getting $\mathrm{H} 1 \mathrm{~N} 1$ vaccine from my university will be painful" ( $\lambda=-.025)$ did not load satisfactorily and was dropped from the analysis. Factor analysis (PCA with Varimax rotation) obtained a single factor solution (eigenvalue $=2.902$, $72.5 \%$ variance; 4 items, $M=2.61, S D=.90, N=198)$. Reliability was good $(\alpha=.87)$.

Subjective Norms. Subjective norms was defined as a measure of family and friends approval of the behavior ${ }^{23}$ and comprised the average of items including: "My immediate family members think I should get the H1N1 flu vaccine at my university this spring $(2010)(\lambda=.69)$,"

205 "I want to do what my immediate family members think I should do about getting the H1N1 flu vaccine this spring (2010)," "My immediate family members would approve of me getting the H1N1 flu vaccine at my university this spring (2010) $(\lambda=.67)$," and "Most of my family members received an H1N1 vaccine last fall (Fall 2009) $(\lambda=.60)$," and similarly for friends (Friends think I should, $\lambda=.67$; friends approve of me getting a vaccine, $\lambda=.58$; friends received 210 a vaccine, $\lambda=.67$ ). Factor analysis (PCA with Varimax rotation) indicated most items loaded acceptably on factor one $(\lambda>.55)$. Two items (I want to do what my immediate family members think..." $\lambda=0.30$; and "I want to do what my friends think..." $\lambda=0.46$ ) did not load satisfactorily were dropped from the study. Factor analysis (PCA with Varimax rotation) obtained a two-factor solution (eigenvalue of first factor $=2.514,41.9 \%$ variance; 6 items, $M=$

$2152.73, S D=.63, N=198)$. Reliability was acceptable $(\alpha=.72)$.

Perceived Behavioral Control. Perceived behavioral control was defined as an individual's perception of their ability to perform a specific behavior. ${ }^{23}$ The scale was assessed as an average of two items including: "It is totally up to me whether I would like to obtain an H1N1 flu vaccine at my university," and "It is mostly up to me to decide whether or not I get an 220 H1N1 flu vaccine at my university" (2 items, $r=.28 ; p<.001, M=4.08, S D=.61, N=196$ ). 
Perceived Comparative Susceptibility. Defined as "the likelihood of experiencing personal harm if no action is taken, ${ }^{, 39,41}$ the three items assessing susceptibility loaded excellently and included: "Compared to the average man/ woman your age, how likely are you to get the H1N1 flu?" $(\lambda=.86)$, "Compared to all students similar to you who did get a flu shot, 225 how likely do you think you are to get the H1N1 flu?" $(\lambda=.88)$, "Compared to all students similar to you who did not get a flu shot, how likely do you think you are to get the H1N1 flu?" $(\lambda=.86)$. Factor analysis (PCA with Varimax rotation) obtained a one-factor solution (eigenvalue of first factor $=2.241,74.7 \%$ variance; 3 items, $M=2.44, S D=.84, N=198$ ). Reliability was acceptable $(\alpha=.83)$.

Side effects. Measured as: "How likely do you think it is that the flu vaccine would cause a person to have a severe reaction?" ( 1 item, $M=1.83, S D=.38, N=198$ ).

Self-efficacy. Understood as the ease or difficulty in performing a behavior in a specific domain, ${ }^{37}$ self-efficacy was measured as the average of two items: "If I take precautions, I will lower my risk of contracting the H1N1 infection enough that I probably don't need to get the

235 H1N1 vaccine anyway," and "If I take all the necessary precautions, I don't need to get the H1N1 vaccine"; $r=.57, p<.001, N=198 ; M=3.49, S D=.90)$.

Behavioral Intention. Defined as whether or not a person plans to perform a particular behavior, ${ }^{23}$ items assessing this measure included the average of: "I intend to get an H1N1 vaccine at my university this season," $(\lambda=.94)$, "I will try to get an H1N1 vaccine at my 240 university this season," $(\lambda=.86)$, and "I plan to get an H1N1 vaccine at my university this season" $(\lambda=.95 ; 3$ items, $M=1.89, S D=.88, N=198)$. Factor analysis (PCA with Varimax 
rotation) obtained a single factor solution (eigenvalue $=2.506,83.5 \%$ variance; 3 items, $M=$ $1.89, S D=.88, N=198)$. Reliability was $\operatorname{good}(\alpha=.90)$.

\section{Multivariate Analysis}

To test the hypotheses, a hierarchical regression model was constructed. Vaccine side effects, self-efficacy, and perceived comparative susceptibility were entered as independent variables (IVs) in the first block. Attitude, subjective norms, and perceived behavioral control were entered together as IVs in the second block. Behavioral intention was entered as the dependent variable (DV, Table 2).

The overall model explained a substantial and significant $51.7 \%(p<.001)$ amount of variance in intentions (Table 2). The first model with side effects, self-efficacy, and perceived comparative susceptibility explained $13.8 \%(p<.001)$ of variance in vaccine intentions. After accounting for side effects, self-efficacy, and perceived comparative susceptibility, the TPB model comprising attitudes, subjective norms, and perceived behavioral control explained an 255 additional $37.9 \%(p<.001)$ of variance in vaccine intentions.

In the first block, self-efficacy made a significant contribution $(b=-.255, p<.001$; Table 2) to intentions after controlling for vaccine side effects and perceived comparative susceptibility. Thus higher self-efficacy predicted lower vaccination intentions and H1a was supported. Perceived comparative susceptibility made a significant contribution to intentions

260 after controlling for vaccine side effects and self-efficacy $(b=0.238, p=.001$, Table 2$)$. Thus H1b was supported.

In the second block, the regression coefficients demonstrate that all TPB predictors, attitudes $(b=.476, p<.001)$, subjective norms $(b=.335, p<.001)$, and perceived behavior 
control $(b=-.306, p<.001)$ were significant contributors to behavioral intention. Attitudes and

265 perceived behavioral control (in the negative direction) are fairly close in strength, while subjective norms are close in its contribution to $\mathrm{A} / \mathrm{H} 1 \mathrm{~N} 1$ vaccination intentions. Thus $\mathrm{H} 2 \mathrm{a}, \mathrm{H} 2 \mathrm{~b}$, and $\mathrm{H} 2 \mathrm{c}$ are supported (Table 2).

\section{Comment}

The study is the first to demonstrate the substantial contribution of the TPB constructs in

270 vaccine intentions for college students who have not previously received a flu vaccine after accounting for the contribution of self-efficacy and perceived comparative susceptibility.

Furthermore, the findings demonstrate that controlling for vaccine side effects, self-efficacy and perceived comparative susceptibility are important factors influencing student vaccination decisions for this segment of students. Because prior behaviors are a significant predictor of

275 future intentions and behaviors, the study makes a unique contribution by identifying salient factors contributing to vaccination intentions for this specific segment of students.

The support for the overall TPB model after accounting for the contribution of selfefficacy and perceived comparative susceptibility suggests important insights for vaccine communication. Because preventive health behaviors for emergent risks are often received with

280 doubts such as those regarding adequate vaccine testing and safety, highlighting positive beliefs of the $\mathrm{A} / \mathrm{H} 1 \mathrm{~N} 1$ vaccine as beneficial, useful, helpful and good can promote vaccination intentions. Second, the study extends prior research finding positive influence of social networks on health behaviors by demonstrating the contribution of subjective norms of friends and family (i.e., whether family and friends think it is good, or have received the vaccine) in this audience segment. Third, independent of the contribution of self-efficacy, vaccine intentions in this group 
of college students is also significantly influenced by perceived behavioral control—-the perception that the vaccination acceptance decision is completely in the hands of the individual. While this is in line with existing findings of resistance to authority in college students in general, it also suggests this audience segment (i.e., those who have not previously accepted a flu 290 vaccine) is significantly influenced by a sense of individual control over decisions.

Furthermore, the study demonstrates support for two clinically significant variables of self-efficacy and perceived comparative susceptibility as important factors to take into consideration. First, self-efficacy is an important contributor in vaccination intentions for students who have not previously obtained a flu vaccine such that students with higher self-

295 efficacy are less likely to plan to obtain the vaccine. Because of challenges inherent in increasing acceptance of voluntary, preventive vaccines in young adults (e.g., a disregard for authoritative recommendations ${ }^{7,8,12}$ ), vaccine communication among students who have not accepted the flu vaccine can highlight how self-efficacy is demonstrated through portraying vaccine acceptance. Second, perceived comparative susceptibility has found support in several risk preventive

300 behaviors and the study findings extend its applicability in vaccination intentions of college students. For this segment of students, the study finds that, controlling for self-efficacy and perception of vaccine side effects, increasing susceptibility compared to other students like them promotes vaccination intentions.

\section{Limitations}

While the study makes an important contribution to the applicability of the TPB to the domain of student flu vaccine intentions, several limitations should be noted. First, because the study design was cross-sectional, the findings are primarily correlational. Second, although the 
convenience in-class recruitment strategy compromised the ecological validity of the study, the study sample is reflective of the overall undergraduate student population of the university.

310 Third, as participants were self-selected into the study, the findings may be biased toward those students who were more (or less) concerned about vaccination due to pre-existing beliefs about vaccination, and non-response bias cannot be assessed. Fourth, since the survey was administered to undergraduate students from classes in the communications department which generally have a greater focus on media effects and health campaigns, care should be taken to

315 not overgeneralize its representativeness to a larger student population. Because studies on college students in general reflect important similarities in beliefs among college students (e.g., in the existence of optimistic bias), future studies can extend the findings among a range of college student demographics. Fifth, although the measures used in the study are from validated scales, these rely on participant self-reports and are susceptible to a recall bias.

\section{Conclusions}

The study demonstrates support for the TPB model in college student vaccination decisions for the specific segment of students who have not received a flu vaccine. Furthermore, for this specific segment of college students, this study is the first to provide insight into the contribution of self-efficacy and perceived comparative susceptibility. This is a particularly

325 challenging group for public health professionals to reach, partly because of the significant influence of past behaviors on future intentions and behaviors. Segmenting audiences helps understand the beliefs and motivations of specific target audiences and is key to the success of health communication efforts seeking to reach population segments that can benefit from a specific health behavior with prevention messages and behavior recommendations. ${ }^{4}$ 


\section{Implications for Student Health Centers}

To reach college students who have not previously received a flu shot, vaccine communication messages should underscore individual choice and ability to obtain the vaccine as an act of responsibility, highlight positive beliefs (e.g., usefulness and benefits), susceptibility compared to other college students, testimony of family and friends, and control factors. 
1. American College Health Association. National College Health Assessment II. Undergraduate Students Reference Group Executive Summary, Spring 2013. Retrieved on February 23, 2014 from http://www.acha-ncha.org/docs/ACHA-NCHAII_UNDERGRAD_ReferenceGroup_ExecutiveSummary_Spring2013.pdf

340 2. American College Health Association. National College Health Assessment II. Spring 2013 Reference Group Executive Summary. Retrieved on February 21, 2014 from http://www.achancha.org/docs/ACHA-NCHA-II_ReferenceGroup_ExecutiveSummary_Spring2013.pdf

3. American College Health Association. Healthy Campus 2020 Student Objectives: Health Impediments to Academic Performance. Retrieved Feb 23, 2014 from

345 www.acha.org/.../docs/HC2020_Student_Objectives_spreadsheet.xls

4. Centers for Disease Control and Prevention. Introduction and biology of influenza: influenza control and prevention recommendations. 2012. Retrieved January 11, 2012 from http://www.cdc.gov/flu/professionals/acip/background.htm.

5. Centers for Disease Control and Prevention. Seasonal influenza (flu): What you should know 350 for the 2013 - 2014 influenza season. Retrieved March 3, 2014 from http://www.cdc.gov/flu/about/season/flu-season-2013-2014.htm

6. World Health Organization (2014). Global alert and response: Current WHO phase of pandemic alert for Pandemic (H1N1) 2009. Retrieved February 23, 2014, from http://www.who.int/csr/disease/swineflu/phase/en/

355 7. Elkind D. Egocentrism in adolescence. Child Dev. 1967; 38: 1025-1034.

8. Elkind D. Cognitive structure and adolescent experience. Adolescence. 1968; 2: 427-434

9. Kim HK, Niederdeppe J. Exploring optimistic bias and the integrative model of behavioral prediction in the context of a campus influenza outbreak. J Health Commun. Epub September 28, 2012. PMID: 23020687.

360 10. Decker JF, Slawson RM. An evaluation of behavioral health compliance and microbial risk factors on student populations within a high-density campus. J Am Coll Health. 2012; 60: 584595.

11. Seale H, Mak JP, Razee H, et al. Examining the knowledge, attitudes, and practices of domestic and international university students toward seasonal and pandemic influenza. BMC

365 Public Health. 2012; 12: 307-312.

12. Byme C, Walsh J, Kola S, Sarma KM. Predicting intention to uptake H1N1 influenza vaccine in a university sample. Br J Health Psychol. 2012; 17(13): 582-595. doi: 10.1111/j.20448287.2011.02057.x.

13. Sellors J, Zimic-Vincetic M, Howard M, et al. Lack of compliance with hepatitis B 370 vaccination among Canadian STD patients: candidates for an accelerated immunization schedule? Can J Public Health. 1997; 88: 210-211. 
14. Centers for Disease Control and Prevention. Interim results: state-specific influenza A (H1N1) 2009 monovalent vaccination coverage_-United States, October 2009_January 2010. MMWR Morb Mortal Wkly Rep. 2010; 59: 363-368. Retrieved on January 11, 2012, from

375 http://www.cdc.gov/mmwr/preview/mmwrhtml/mm5912a2.htm.

15. Friedman AL, Shepeard H. Exploring the knowledge, attitudes, beliefs, and communication preferences of the general public regarding HPV: findings from CDC focus group research and implications for practice. Health Educ Behav. 2007; 34: 471-485.

16. Lee GM, Greene SK, Weintraub ES, et al. H1N1 and seasonal influenza vaccine safety in the vaccine safety datalink project. Am J Prev Med. 2011; 41(2): 121-128. doi: 10.1016/j.amepre.2011.04.004.

17. Byrne C, Walsh J, Kola S, et al. Predicting intention to uptake H1N1 influenza vaccine in a university sample. Br J Health Psychol. 2012; 17: 582-595.

18. Renner B, Reuter T. Predicting vaccination using numerical and affective risk perceptions: 385 the case of A/H1N1 influenza. Vaccine. 2012; 30: 7019-7026.

19. Stroud C, Nadig L, Altevogt BM. The 2009 H1N1 influenza vaccination campaign: summary of a workshop series. Forum on medical and public health preparedness for catastrophic events, Board on Health Sciences Policy. Washington, DC: The National Academies Press; 2010.

20. Nyhan B, Reifler J, Richey S, et al. The role of social networks in influenza vaccine attitudes 390 and intentions among college students in the Southeastern United States. J Adolesc Health. 2012; 51(3): 302-304. doi: 10.1016/j.jadohealth.2012.02.014.

21. Frew PM, Painter JE, Hixson B, et al. Factors mediating seasonal and influenza A (H1N1) vaccine acceptance among ethnically diverse populations in the urban south. Vaccine. 2012; 30(28): 4200-4208. doi: 10.1016/j.vaccine.2012.04.053.

395 22. Cornally N, Ann Deasy E, McCarthey G. Student nurses' intention to get the influenza vaccine. Br J Nurs. 2013; 22(1): 1207-1211.

23. Ajzen I. Constructing a TpB questionnaire: Conceptual and methodological considerations. 2002. Available from http://people.umass.edu/aizen/pdf/tpb.measurement.pdf

24. Armitage CJ, Conner M. Distinguishing perceptions of control from self-efficacy: Predicting 400 consumption of a low-fat diet using the theory of planned behavior. J Appl Soc Psychol. 1999; 29(1): 72-90. doi: 10.1111/j.1559-1816.1999.tb01375.x.

25. Liao Q, Cowling BJ, Lam WW, et al. Factors affecting intention to receive and self-reported receipt of 2009 pandemic H1N1 vaccine in Hong Kong: a longitudinal study. PLoS One. 2011; e17713. doi: 10.1371/journal.pone.0017713.

405 26. Myers LB, Goodwin R. Determinants of adults' intention to vaccinate against pandemic swine flu. BMC Public Health. 2011; 11. doi: 10.1186/1471-2458-11-15. Available from: http://www.biomedcentral.com/1471-2458/11/15. 
27. Painter JE, Gargano LM, Sales JM, et al. Correlates of 2009 H1N1 influenza vaccine acceptability among parents and their adolescent children. Health Educ Res. 2011; 26: 751-760.

410 28. Myers LB, Goodwin R. Using a theoretical framework to determine adults' intention to vaccinate against pandemic swine flu tin priority groups in the UK. Public Health. 2012; 126(S1): S53-S56. Doi: 10.1016/j.puhe.2012.05.024.

29. Masser BM, White KM, Hamilton K, McKimmie BM. An examination of the predictors of blood donors' intentions to donate during two phases of an avian influenza outbreak.

415 Transfusion. 2011; 51(3): 548-557. doi: 10.1111/j.1537-2995.2010.02887.x.

30. Clowes R, Masser BM. Right here, right now: The impact of the blood donation context on anxiety, attitudes, subjective norms, self-efficacy, and intention to donate blood. Transfusion. 2012; 52(7): 1560-1565. doi: 10.1111/j.1537-2995.2011.03486.x.

31. Sandberg T, Conner M. Anticipated regret as an additional predictor in the theory of planned 420 behavior: A meta-analysis. Br J Soc Psychol. 2008; 47(Pt 4): 589-606.

32. Kroshus E, Baugh CM, Daneshvar DH, Viswanath K. Understanding concussion reporting using a model based on the theory of planned behavior. J Adolesc Health. 2014; 54(3): 269-274. doi: 0.1016/j.jadohealth.2013.11.011.

33. Cafiero M. Nurse practitioners' knowledge, experience, and intention to use health literacy 425 strategies in clinical practice. J Health Commun. 2013; 18(Suppl. 2013):70-81. doi: 10.1080/10810730.2013.825665.

34. Deskins S, Harris CV, Bradlyn AS, et al. Preventive care in Appalachia: Use of the theory of planned behavior to identify barriers to participation in cholesterol screenings among West Virginians. J Rural Health. 2006; 22(4):367-374.

430 35. Ross LT, Ross TP, Farber S, Davidson C, Trevino M, Hawkins A. The theory of planned behavior and helmet use among college students. Am J Health Behav. 2011;35(5):581-590.

36. Ajzen I. The theory of planned behavior. Organ Behav Hum Decis Process. 1991; 50:179211.

37. Ajzen I. Perceived behavioral control, self-efficacy, locus of control, and the theory of 435 planned behavior. J App Soc Psychol. 2006; 32(4): 665-683. doi: 10.1111/j.15591816.2002.tb00236.x.

38. Just the Facts (2009-2010). University of Louisville, Ky. Retrieved March $7^{\text {th }}, 2014$, from https://louisville.edu/institutionalresearch/files/just-the-facts/jtf200910.pdf

39. McQueen A, Swank PR, Bastian LA, et al. Predictors of perceived susceptibility of breast cancer and changes over time: a mixed modeling approach. Health Psychol. 2008; 27: 68-77.

40. Chapman GB, Coups EJ. Predictors of influenza vaccine acceptance among Healthy adults. Prev Med. 1999; 29(4): 249-262. doi: http://dx.doi.org/10.1006/pmed.1999.0535.

41. Weinstein ND. Optimistic biases about personal risks. Science. 1989; 246:1232-1233. 